JOURNAL OF

FUNCTION SPACES AND APPLICATIONS

Volume 7, Number 3 (2009), 241-250
(C) 2009, Scientific Horizon http://www.jfsa.net

\title{
The boundedness of commutator of Riesz transform associated with Schrödinger operators on a Hardy space
}

\author{
Canqin Tang ${ }^{1}$ and Chuanmei Bi \\ (Communicated by Vladimir Maz'ya)
}

2000 Mathematics Subject Classification. Primary: 42B20, 42B30.

Keywords and phrases. Commutator, BMO, Riesz transform, Schrödinger operators, Hardy space.

Abstract. In this paper, we study the boundedness of commutator $[b, T]$ of Riesz transform associated with Schrödinger operator and $b$ is $B M O$ type function, note that the kernel of $T$ has no smoothness, and the boundedness from $H_{b}^{1}\left(R^{n}\right) \rightarrow L^{1}\left(R^{n}\right)$ is obtained.

\section{Introduction}

It is well know that the Calderón-Zygmmund singular operator is an important operator in Harmonic Analysis. The properties of the C-Z singular operator and its commutator are studied by many scholars. Such as in [1] [2] [3]. Among this, C. Perez [3] states the $H_{b}^{1}\left(R^{n}\right) \rightarrow L^{1}\left(R^{n}\right)$ boundedness of the commutator $[b, T]$, where $T$ is a C-Z singular operator and $b \in B M O\left(R^{n}\right)$.

Schrödinger differential operator is another interesting topic in Harmonic Analysis. Let $A=-\Delta+V(x)$ be the Schrödinger differential operator

\footnotetext{
${ }^{1}$ This work is partially supported by NNSF 107010178
} 
on $R^{n}, n \geq 3$. Throughout the paper we will assume that $V(x)$ is a non-zero, nonnegative potential, and belongs to $B_{q}$ for some $q>n / 2$. Let $T=\nabla(-\Delta+V(x)),[b, T] f=b T f-T b f$. The $L^{p}$ boundedness of $T$ and the commutator $[b, T]$ is widely studied in [4] [5] when $V(x)$ satisfies some conditions. The basic idea in [4] is to find a pointwise estimate of the kernel and the comparison to the kernel of classical Riesz transform. But in [6], Z. Guo, P. Li and L. Peng adopt a different idea to get the $L^{p}$ boundedness of some commutators of Riesz transforms associated to Schrödinger operator since the kernel no longer satisfied the regular condition of Calderón-Zygmmund kernel. Note that the kernels have some other kind of smoothness $H(m)$. Inspired by their work, we will consider $H_{b}^{1}\left(R^{n}\right) \rightarrow L^{1}\left(R^{n}\right)$ boundedness of commutator $[b, T]$ in this case, where $b \in B M O$.

\section{Some preliminaries and notations}

In this section, we first recall some definitions and lemmas we need in this paper.

$Q$ will always denote a cube with sides parallel to the axes. $\lambda Q(\lambda>0)$ denotes the cube has the same center as $Q$ and dilated by $\lambda$. Also $B=B\left(x_{B}, r\right)$ will denote a ball centered at $x_{B}$ with radius $r$ and corresponding notation applies for $\lambda B$. We adopt the idea of Strömberg. Recall that the sharp function of Fefferman-Stein is defined by

$$
M^{\sharp} f(x)=\sup _{x \in B} \frac{1}{|B|} \int_{B}\left|f(y)-f_{B}\right| d y,
$$

simultaneity, recall that $B M O$ is defined by

$$
B M O\left(R^{n}\right)=\left\{f \in L_{l o c}^{1}\left(R^{n}\right):\|f\|_{B M O}=\left\|M^{\sharp} f\right\|_{\infty}<\infty\right\} .
$$

where $f_{B}=\frac{1}{|B|} \int_{B} f(y) d y$, and the supremum is taken on all balls $B$ with $x \in B$. Two basic facts about $B M O$ will be used in this paper,

$$
\left|f_{2^{k} B}-f_{B}\right| \leq C(k+1)\|f\|_{B M O}, k>0
$$

and the one due to John-Nirenberg

$$
\|f\|_{B M O} \sim \sup _{B}\left(\frac{1}{|B|} \int_{B}\left|f(y)-f_{B}\right|^{p} d y\right)^{1 / p}, p>1
$$


In this paper we will assume that $V$ belongs to $B_{q}$ for some $q>\frac{n}{2}$, that is,

$$
\left(\frac{1}{|B|} \int_{B} V^{q}(x) d x\right)^{1 / q} \leq C\left(\frac{1}{|B|} \int_{B} V(x) d x\right),
$$

for every ball $B \subset \mathbb{R}^{n}$. Define auxiliary function

$$
\rho(x, V)=\rho(x)=\frac{1}{m(x, V)}=\sup \left\{r>0: \frac{1}{r^{n-2}} \int_{B(x, r)} V(y) d y \leq 1\right\} .
$$

Definition 2.1. ([3]) A function $a$ is a $b$ - atom if there is a cube $Q$ for which

$$
\text { (1) } \operatorname{supp} a \subset Q,(2)\|a\|_{L^{\infty}} \leq \frac{1}{|Q|},(3) \int_{Q} a(y) d y=0, \text { (4) } \int_{Q} a(y) b(y) d y=0 \text {. }
$$

The space $H_{b}^{1}\left(\mathbb{R}^{n}\right)$ consists of the subspace of $L^{1}\left(\mathbb{R}^{n}\right)$ functions $f$ which can be written as $f=\sum_{j} \lambda_{j} a_{j}$ where $a_{j}$ are $b$ - atom and $\lambda_{j}$ are complex numbers with $\sum_{j}\left|\lambda_{j}\right|<\infty$ and define its space norm as $\|f\|_{H_{b}^{1}}=\inf \left(\sum_{j}\left|\lambda_{j}\right|\right)$. Like the definition in [6], in our problem, we need the following smoothness of kernel.

Definition 2.2. $K(x, y)$ is said to satisfy $H(m)$ for some $m>1$, if there exist a constant $C$ such that, for any $l>0, y, x_{B} \in R^{n}$ with $\left|y-x_{B}\right| \leq l$, then

$$
\sum_{k=5}^{\infty} k\left(2^{k} l\right)^{n / m^{\prime}}\left(\int_{2^{k} l \leq\left|x-x_{B}\right|<2^{k+1} l}\left|K(x, y)-K\left(x, x_{B}\right)\right|^{m} d x\right)^{1 / m} \leq C,
$$

where $1 / m^{\prime}=1-1 / m$.

It is easy to prove that if $K(x, y)$ is the usual Calderón-Zygmund kernel, it satisfies $H(m)$ for any $m \geq 1$.

Lemma A. ([4]) Suppose $V \in B_{q_{0}}, q_{0}>1$. Assume that $-\Delta u+(V(x)+$ $i \tau) u=0$ in $B\left(x_{0}, 2 R\right)$ for some $x_{0} \in R^{n}, R>0$. Then

(a) for $x \in B\left(x_{0}, R\right)$,

$|\nabla u(x)| \leq C \sup _{B\left(x_{0}, 2 R\right)}|u| \cdot \int_{B\left(x_{0}, 2 R\right)} \frac{V(y)}{|x-y|^{n-1}} d y+\frac{C}{R^{n+1}} \int_{B\left(x_{0}, 2 R\right)}|u(y)| d y$,

(b) if $(n / 2)<q_{0}<n,(1 / t)=\left(1 / q_{0}\right)-(1 / n), k_{0}>\log _{2} C_{0}+1$, then 


$$
\left(\int_{B\left(x_{0}, R\right)}|\nabla u|^{t} d x\right)^{1 / t} \leq C R^{\left(n / q_{0}\right)-2}\left\{1+R m\left(x_{0}, V\right)\right\}^{k_{0}} \sup _{B\left(x_{0}, 2 R\right)}|u| .
$$

Lemma B. ([6]) Suppose $V \in B_{q}$ for some $q>n / 2$. Let $N>$ $\log _{2} C_{0}+1$, where $C_{0}$ is the constant in doubling measure inequality $\int_{B(x, 2 r)} V(y) d y \leq C_{0} \int_{B(x, r)} V(y) d y$. Then for any $x_{0} \in R^{n}, R>0$,

$$
\frac{1}{\left\{1+m\left(x_{0}, V\right) R\right\}^{N}} \int_{B\left(x_{0}, R\right)} V(\xi) d \xi \leq C R^{n-2}
$$

Let $\Gamma(x, y, \tau)$ denote the fundamental solution for the Schrödinger operator $-\Delta+(V(x)+i \tau)$. A pointwise estimate of $\Gamma(x, y, \tau)$ given in $[4]$ is a key result to our calculus.

Theorem A. ([4]) Suppose $V \in B_{n / 2}$. Then, for any $x, y \in R^{n}, \tau \in R$, and integer $k>0$,

$$
\Gamma(x, y, \tau) \leq \frac{C_{k}}{\left\{1+|\tau|^{1 / 2}|x-y|\right\}^{k}\{1+m(x, V)|x-y|\}^{k}} \cdot \frac{1}{|x-y|^{n-2}} .
$$

\section{Main results and proofs}

Let $T=\nabla(-\Delta+V)^{-1 / 2}$, we'll study the boundedness of commutator of $T$ in Hardy space.

Theorem 3.1. Let $V \in B_{q}$ and $n / 2<q<n, b \in B M O$. Then $[b, T]$ is a bounded operator from $H_{b}^{1}\left(R^{n}\right)$ to $L^{1}\left(R^{n}\right)$.

To prove Theorem 3.1, we need the following lemmas.

Lemma 3.1. Let $T=\nabla(-\Delta+V)^{-1 / 2}, K(x, y)$ be kernel of $T$. Suppose $V \in B_{q}$ for some $n / 2<q<n$. Then there exist $\delta>0$ and for any integer $k>0,0<h<|x-y| / 16$, 
(1)

$$
\begin{aligned}
&|K(x, y)| \leq \frac{C_{k}}{\{1+m(y, V)|x-y|\}^{k}} \cdot \frac{1}{|x-y|^{n-1}} \\
& \times\left(\int_{B(y,|x-y|)} \frac{V(\xi)}{|x-\xi|^{n-1}} d \xi+\frac{1}{|x-y|}\right) \\
& \quad \times\left(\int_{B(y,|x-y|)} \frac{V(\xi)}{|x-\xi|^{n-1}} d \xi+\frac{1}{|x-y|}\right) .
\end{aligned}
$$

Proof. By partial integral, we know that

$$
K(x, y)=-\frac{1}{2 \pi} \int_{R}(-i \tau)^{-1 / 2} \nabla_{x} \Gamma(x, y, \tau) d \tau .
$$

Fix $x, y \in R^{n}, \quad R=\frac{|x-y|}{8}, 1 / t=1 / q-1 / n, \delta=2-n / q>0$, $0<h<R / 2$, we have

$$
|K(x, y+h)-K(x, y)| \leq \frac{1}{2 \pi} \int_{R}|\tau|^{-1 / 2}\left|\nabla_{x} \Gamma(x, y+h, \tau)-\nabla_{x} \Gamma(x, y, \tau)\right| d \tau .
$$

It follows from the imbedding theorem of Morrey and Lemma A(b) that

$$
\begin{aligned}
& \left|\nabla_{x} \Gamma(x, y+h, \tau)-\nabla_{x} \Gamma(x, y, \tau)\right| \\
& \quad \leq C|h|^{1-n / t}\left(\int_{B(x, R)}\left|\nabla_{y} \nabla_{x} \Gamma(x, z, \tau)\right|^{t} d z\right)^{1 / t} \\
& \quad \leq C|h|^{1-n / t} R^{n / q-2}\{1+R m(y, V)\}^{R_{0}} \sup _{z \in B(y, 2 R)}\left|\nabla_{x} \Gamma(x, z, \tau)\right| .
\end{aligned}
$$

Since $\Gamma(x, z, \tau)=\Gamma(z, x,-\tau)$, we have $\nabla_{x} \Gamma(x, z, \tau)=\nabla_{y} \Gamma(z, x-\tau)$. It follows from Lemma A(a) that,

$$
\begin{aligned}
\sup _{z \in B(y, 2 R)} & \left|\nabla_{x} \Gamma(x, z, \tau)\right| \leq \sup _{z \in B(y, 2 R)}\left|\nabla_{y} \Gamma(z, x,-\tau)\right| \\
\leq & \sup _{z \in B(y, 2 R)}\left\{\sup _{\eta \in B(z,|z-x| / 4)}|\Gamma(\eta, y,-\tau)| \int_{B(z,|z-x| / 2)} \frac{V(\xi)}{|z-\xi|^{n-1}} d \xi\right. \\
& \left.+\frac{C}{|z-x|^{n+1}} \int_{B(z,|z-x| / 2)} \Gamma(\xi, x,-\tau) d \xi\right\}
\end{aligned}
$$


Also from Theorem A, [4, Lemma 1.4(b)], using the fact that $|\eta-x| \sim$ $|z-x|,|\xi-x| \sim|z-x|,|z-x| \sim|x-y|,|z-\xi| \sim|x-\xi|$ and choosing $k_{1}$ sufficiently large, we obtain

$$
\begin{aligned}
& \sup _{z \in B(y, 2 R)}\left|\nabla_{x} \Gamma(x, z, \tau)\right| \\
\leq & \sup _{z \in B(y, 2 R)} \frac{C_{k_{1}}}{\left\{1+|\tau|^{1 / 2}|\eta-x|\right\}^{k_{1}}\{1+m(\eta, V)|\eta-x|\}^{k_{1}}} \\
& \times \frac{1}{|\eta-x|^{n-2}} \int_{B(z,|z-x| / 2)} \frac{V(\xi)}{|z-\xi|^{n-1}} d \xi \\
& +\frac{C_{k_{1}}}{|z-x|^{n+1}} \int_{B(z,|z-x| / 2)} \frac{C_{k_{1}}}{\left\{1+|\tau|^{1 / 2}|\xi-x|\right\}^{k_{1}}\{1+m(\xi, V)|\xi-x|\}^{k_{1}}} \\
& \times \frac{1}{|\xi-x|^{n-2}} \\
& z \in B(y, 2 R) \\
\quad & \times \frac{1}{|x-y|^{n-2}} \int_{B(y,|x-y|)} \frac{C_{k_{1}}}{|x-\xi|^{n-1}} d \xi \\
& +\frac{C_{k_{1}}}{|x-y|^{n-1}} \cdot \frac{C_{k_{1}}}{\left\{1+|\tau|^{1 / 2}|x-y|\right\}^{k_{1}}\{1+m(y, V)|x-y|\}^{k_{1}}} .
\end{aligned}
$$

Computing as in the proof of Lemma 4 in [6], the assertion is proved.

Lemma 3.2. Let $T=\nabla(-\Delta+V)^{-1 / 2}, V \in B_{q}$ for some $n / 2<q<n$. $K(x, y)$ be a kernel of $T$. Then $K(x, y)$ satisfies $H(m)$, where $1 / m=$ $1 / q-1 / n$.

Proof. For any $l>0, y, x_{B} \in R^{n}$ with $\left|y-x_{B}\right| \leq l$, choosing $N$ sufficiently large, by Lemma $\mathrm{B},(3.1)$ and $V \in B_{q}$, we have

$$
\begin{aligned}
& \left(\int_{2^{k} l \leq\left|x-x_{B}\right|<2^{k+1} l}\left|K(x, y)-K\left(x, x_{B}\right)\right|^{m} d x\right)^{1 / m} \\
\leq & \left(\int_{2^{k} l \leq\left|x-x_{B}\right|<2^{k+1} l} \mid \frac{C_{N}}{\left\{1+m\left(x_{B}, V\right)\left|x-x_{B}\right|\right\}^{N}}\right. \\
& \left.\times\left.\frac{\left|y-x_{B}\right|^{\delta}}{\left|x-x_{B}\right|^{n-1+\delta}}\left(\int_{B(x,|x-y|)} \frac{V(\xi)}{|x-\xi|^{n-1}} d \xi+\frac{1}{\left|x-x_{B}\right|}\right)\right|^{m} d x\right)^{1 / m}
\end{aligned}
$$




$$
\begin{array}{lll}
\leq \quad & C_{N} \frac{l^{\delta}}{\left(2^{k} l\right)^{n-1+\delta}} \cdot \frac{1}{\left\{1+m\left(x_{B}, V\right) 2^{k} l\right\}^{N}}\left\|\int \frac{V(\xi) \chi_{B\left(x_{B}, 2^{k+3} l\right)}}{|x-\xi|^{n-1}} d \xi\right\|_{L_{x}^{m}} \\
& +\frac{l^{\delta}}{\left(2^{k} l\right)^{n / m^{\prime}+\delta}} \\
\leq \quad & C_{N} \frac{l^{\delta}}{\left(2^{k} l\right)^{n-1+\delta}} \cdot \frac{1}{\left\{1+m\left(x_{B}, V\right) 2^{k} l\right\}^{N}}\left(\int_{B\left(x_{B}, 2^{k+3} l\right)} V(\xi)^{q} d \xi\right)^{1 / q} \\
& +\frac{l^{\delta}}{\left(2^{k} l\right)^{n / m^{\prime}+\delta}} \\
& C_{N} \frac{l^{\delta}}{\left(2^{k} l\right)^{n-1+\delta}} \cdot \frac{1}{\left\{1+m\left(x_{B}, V\right) 2^{k} l\right\}^{N}}\left(\int_{B\left(x_{B}, 2^{k+3} l\right)} V(\xi) d \xi\right) \cdot\left(2^{k} l\right)^{-n / q^{\prime}} \\
& +\frac{l^{\delta}}{\left(2^{k} l\right)^{n / m^{\prime}+\delta}} \\
\leq \quad & C_{N} \frac{l^{\delta}}{\left(2^{k} l\right)^{n-1+\delta}}\left(2^{k} l\right)^{n / q-2}+\frac{l^{\delta}}{\left(2^{k} l\right)^{n / m^{\prime}+\delta}} \\
\leq \quad & C \frac{l^{\delta}}{\left(2^{k} l\right)^{n / m^{\prime}+\delta}} .
\end{array}
$$

Here we have used the fact that $B(x,|x-y|) \subset B\left(x_{B}, 2^{k+3} l\right)$. In fact, for all $\xi \in B(x,|x-y|),\left|\xi-x_{B}\right| \leq|\xi-x|+\left|x-x_{B}\right| \leq|x-y|+\left|x-x_{B}\right| \leq$ $\left|x-x_{B}\right|+\left|y-x_{B}\right|+\left|x-x_{B}\right| \leq 2^{k+3}$. Therefore,

$$
\sum_{k=5}^{\infty} k\left(2^{k} l\right)^{n / m^{\prime}}\left(\int_{2^{k} l \leq\left|x-x_{B}\right|<2^{k+1} l}\left|K(x, y)-K\left(x, x_{B}\right)\right|^{m} d x\right)^{1 / m} \leq \sum_{k=5}^{\infty} \frac{C k}{\left(2^{k}\right)^{\delta}} \leq C
$$

and we are done.

Proof of Theorem 3.1. Let $b \in B M O$. By the atomic decomposition of Hardy space, we only need to prove that there exists a constant $C$ such that for each $b$-atom $a$

$$
\int_{R^{n}}|[b, T] a(y)| d y \leq C\|b\|_{B M O}\|a\|_{H_{b}^{1}\left(R^{n}\right)} .
$$

Suppose $\operatorname{supp} a \subset B\left(x_{B}, l\right)$ for some ball $B$. Then

$$
\int_{R^{n}}|[b, T] a(y)| d y=\int_{2 B}|[b, T] a(y)| d y+\int_{R^{n} \backslash 2 B}|[b, T] a(y)| d y=\mathrm{I}+\mathrm{II} .
$$


The estimate of I follows by the boundedness of $[b, T]$ on $L^{2}\left(R^{n}\right)$ (see [5]) and the size condition of atom $a$, i.e.,

$$
\begin{aligned}
\mathrm{I} & \leq C|B|\left(\frac{1}{|2 B|} \int_{2 B}|[b, T] a(y)|^{2} d y\right)^{1 / 2} \leq C\|b\|_{B M O}|B| \cdot\left(\frac{1}{|B|} \int_{B}|a(y)|^{2} d y\right)^{1 / 2} \\
& \leq C\|b\|_{B M O}|B| \cdot\|a\|_{\infty} \leq C\|b\|_{B M O} .
\end{aligned}
$$

To estimate II, we split $[b, T]$ as $[b, T] a=\left(b-b_{B}\right) T a-T\left(\left(b-b_{B}\right) a\right)$.

Then

$$
\mathrm{II} \leq \int_{R^{n} \backslash 2 B}\left|\left(b(x)-b_{B}\right) T a(x)\right| d x+\int_{R^{n} \backslash 2 B}\left|T\left(\left(b-b_{B}\right) a\right)(x)\right| d x=\mathrm{III}+\mathrm{IV} .
$$

By Lemma 3.2 and cancelation condition, $\int_{B} a(y) d y=0$, so that

$$
\begin{aligned}
& \mathrm{III}=\int_{R^{n} \backslash 2 B}\left|\left(b(x)-b_{B}\right) T a(x)\right| d x \\
& \leq \int_{R^{n} \backslash 2 B}\left|\left(b(x)-b_{B}\right) \int_{B} a(y)\left(K(x, y)-K\left(x, x_{B}\right)\right) d y\right| d x \\
& \leq \int_{B} \int_{R^{n} \backslash 2 B}\left|\left(b(x)-b_{B}\right) a(y)\left(K(x, y)-K\left(x, x_{B}\right)\right)\right| d x d y \\
& \leq \int_{B} \sum_{k=1}^{\infty} \int_{2^{k} l \leq\left|x-x_{B}\right|<2^{k+1} l}\left|\left(b(x)-b_{B}\right) a(y)\left(K(x, y)-K\left(x, x_{B}\right)\right)\right| d x d y \\
& \leq \int_{B} \sum_{k=1}^{\infty}\left(2^{k} l\right)^{n / p} k\left(\int_{2^{k} l \leq\left|x-x_{B}\right|<2^{k+1} l}\left|K(x, y)-K\left(x, x_{B}\right)\right|^{q} d x\right)^{1 / q} \\
& \times \frac{1}{\left(2^{k} l\right)^{n / p} k}\left(\int_{2^{k} l \leq\left|x-x_{B}\right|<2^{k+1} l}\left|b(x)-b_{B}\right|^{p} d x\right)^{1 / p}|a(y)| d y \\
& \leq C \sup _{k \geq 1} \frac{1}{k} \int_{B}|a(y)| d y \cdot \frac{1}{\left(2^{k} l\right)^{n / p}}\left(\int_{2^{k} l \leq\left|x-x_{B}\right|<2^{k+1} l}\left|b(x)-b_{B}\right|^{p} d x\right)^{1 / p} \\
& \leq \quad C \sup _{k \geq 1} \frac{1}{k} \int_{B}|a(y)| d y \\
& \times\left(\frac{1}{\left(2^{k+1} l\right)^{n}} \int_{B\left(x_{B}, 2^{k+1} l\right)}\left|b(x)-b_{2^{k+1} B}+b_{2^{k+1} B}-b_{B}\right|^{p} d x\right)^{1 / p}
\end{aligned}
$$




$$
\begin{aligned}
\leq & C \sup _{k \geq 1} \frac{1}{k} \int_{B}|a(y)| d y \\
& \times\left\{\left(\frac{1}{\left(2^{k+1} l\right)^{n}} \int_{B\left(x_{B}, 2^{k+1} l\right)}\left|b(x)-b_{2^{k+1} B}\right|^{p} d x\right)^{1 / p}+\left|b_{2^{k+1} B}-b_{B}\right|\right\} \\
\leq & C \sup _{k \geq 1} \frac{1}{k} \int_{B}|a(y)| d y \cdot(k+2)\|b\|_{B M O} \\
\leq & C\|b\|_{B M O},
\end{aligned}
$$

where $1 / p+1 / q=1$.

By the definition of $a$, we have

$$
\int_{B}\left(b(y)-b_{B}\right) a(y) d y=\int_{B} a(y) b(y) d y-b_{B} \int_{B} a(y)=0,
$$

For IV, by Lemma 3.2 and Hölder inequality, we have

$$
\begin{aligned}
\mathrm{IV} & =\int_{R^{n} \backslash 2 B}\left|T\left(\left(b-b_{B}\right) a\right)(x)\right| d x \\
= & \int_{R^{n} \backslash 2 B}\left|\int_{B} K(x, y)\left(b(y)-b_{B}\right) a(y) d y\right| d x \\
\leq & \int_{R^{n} \backslash 2 B}\left|\int_{B}\left(K(x, y)-K\left(x, x_{B}\right)\right)\left(b(y)-b_{B}\right) a(y) d y\right| d x \\
\leq & \left.\int_{B} \mid b(y)-b_{B}\right)|| a(y)\left|\int_{R^{n} \backslash 2 B}\right| K(x, y)-K\left(x, x_{B}\right) \mid d x d y \\
\leq & \int_{B}\left|b(y)-b_{B}\right||a(y)| \sum_{k=1}^{\infty} \int_{2^{k} l \leq\left|x-x_{B}\right|<2^{k+1} l}\left|K(x, y)-K\left(x, x_{B}\right)\right| d x d y \\
\leq & \int\left|b(y)-b_{B}\right||a(y)| \sum_{k=1}^{\infty}\left(\int_{2^{k} l \leq\left|x-x_{B}\right|<2^{k+1} l}\left|K(x, y)-K\left(x, x_{B}\right)\right|^{p} d x\right)^{1 / p} \\
& \times\left(\int_{2^{k} l \leq\left|x-x_{B}\right|<2^{k+1} l} d x\right)^{1 / q} d y
\end{aligned}
$$




$$
\begin{aligned}
\leq & \int_{B}\left|b(y)-b_{B}\right||a(y)| \sum_{k=1}^{\infty} k\left(2^{k} l\right)^{n / q} \\
& \times\left(\int_{2^{k} l \leq\left|x-x_{B}\right|<2^{k+1} l}\left|K(x, y)-K\left(x, x_{B}\right)\right|^{p} d x\right)^{1 / p} \cdot \frac{1}{k} d y \\
\leq & C \sup _{k \geq 1} \frac{1}{k} \cdot \frac{1}{|B|} \int_{B}\left|b(y)-b_{B}\right| d y \leq C\|b\|_{B M O} .
\end{aligned}
$$

This is the proof of Theorem 3.3.

Acknowledgment. The authors wish to express their deep thanks to the referee for many valuable comments.

\section{References}

[1] A.P. Calderón and A. Zygmund, On singular integrals, Amer. J. Math., 78 (1956), 85-139.

[2] A.P. Calderón, Commutators of singular integral operators, Proc. Nat. Acad. Sci. USA, 53 (1965), 1092-1099.

[3] T.D. Chu, Boundedness Of commutators associated with Schrödinger operator and fourier multiplier, Master Thesis, HuNan University, China, 2006.

[4] Z. Guo, P. LI and L. Peng, $L^{p}$ boundedness of commutators of Reisz transform associated to Schrödinger operators, http://aps.arxiv.org/abs/0802.3128.

[5] C. Perez, Endpoint estimates for commutators of singular integral operators, J. Function Anal., 128 (1995), 163-185.

[6] Z. Shen, $L^{p}$ estimates for Schrödinger operators with certain potentials, Ann. Inst. Fourier (Grenoble), 45 (1995), 143-176.

Department of Mathematics

Dalian Maritime University

Dalian, Liaoning

P. R. China, 116026

(E-mail : tangcq2000@yahoo.com.cn)

(Received : April 2008) 


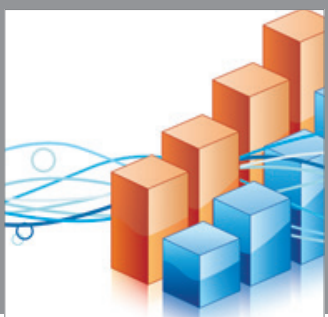

Advances in

Operations Research

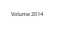

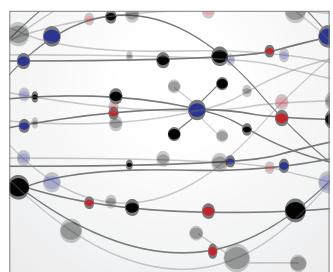

\section{The Scientific} World Journal
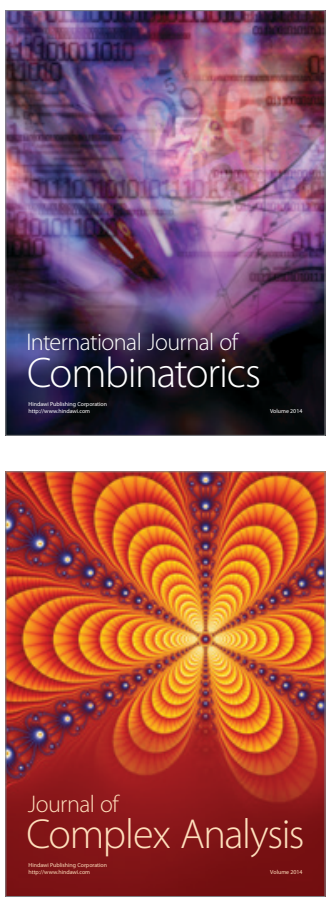

International Journal of

Mathematics and

Mathematical

Sciences
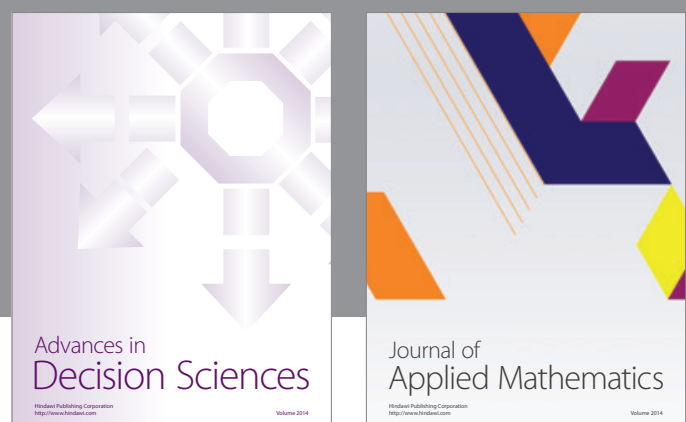

Journal of

Applied Mathematics
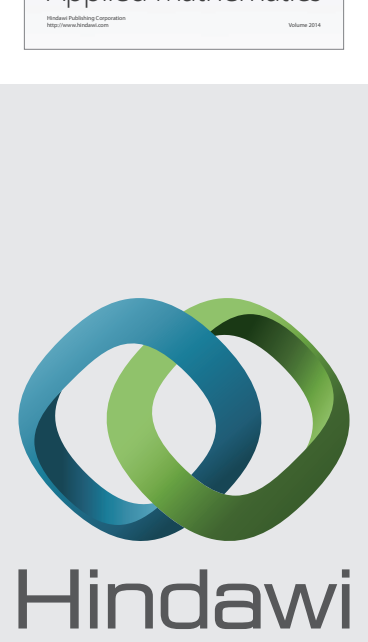

Submit your manuscripts at http://www.hindawi.com
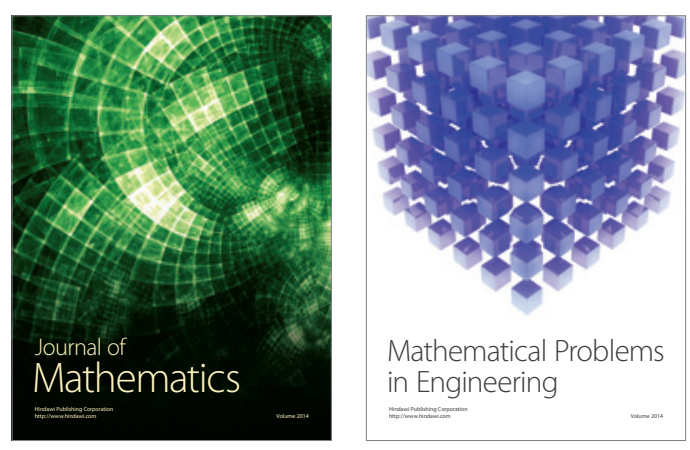

Mathematical Problems in Engineering
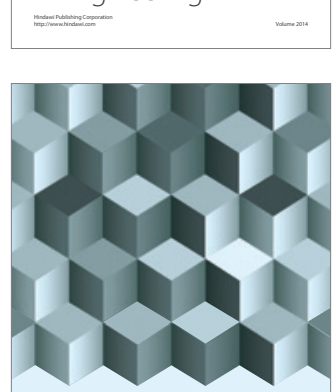

Journal of

Function Spaces
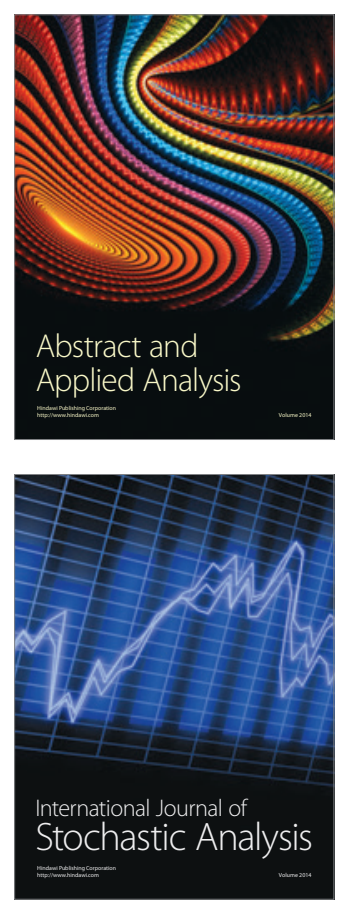

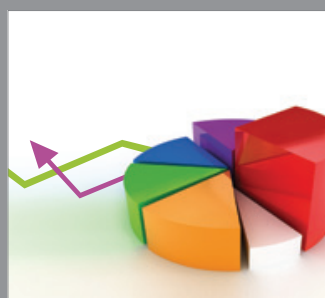

ournal of

Probability and Statistics

Promensencen
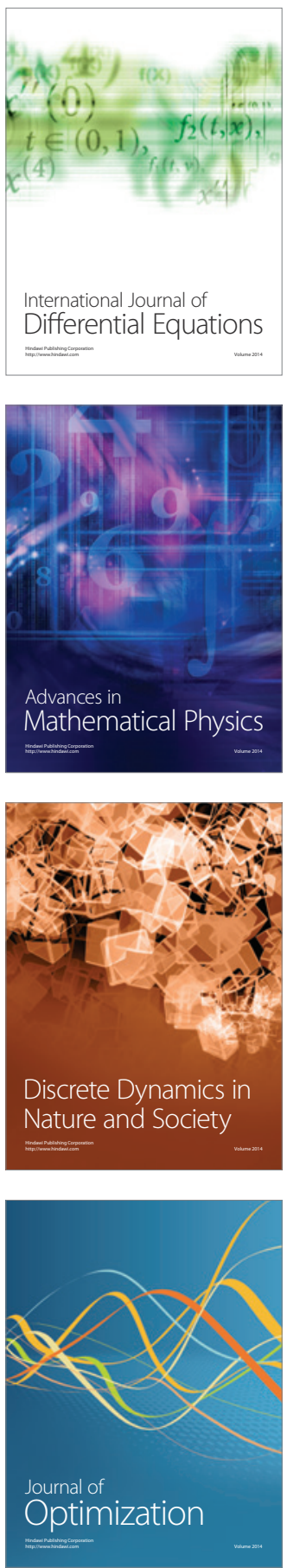